\title{
A Hypothesis on the Possible Potential of Plantago Major in the Treatment of Urticaria
}

\author{
Mohammad Ali Yazdian ${ }^{1}$, Mahmood Khodadoost $^{1}$, Mehdi Gheisari² $^{2}$, Mohammad Kamalinejad ${ }^{3}$, \\ Amir Hooshang Ehsani ${ }^{4}$, Behrooz Barikbin ${ }^{5}$
}

\author{
${ }^{1}$ Department of Traditional Medicine, Shahid Beheshti University of Medical Sciences, Tehran, Iran. \\ ${ }^{2}$ Department of Dermatology, Shahid Beheshti University of medical sciences, Tehran, Iran. \\ ${ }^{3}$ Department of Pharmacology, Shahid Beheshti University of medical sciences, Tehran, Iran. \\ ${ }^{4}$ Department of Dermatology, Tehran University of medical sciences, Tehran, Iran. \\ ${ }^{5}$ Laser Application in Medical Sciences Research Center, Shahid Beheshti University of Medical Sciences, Tehran, Iran.
}

\begin{abstract}
Background:Urticaria is a skin disease characterized by pruritic edematous lesions also called wheals. Although these lesions are self-limited they may result in lowered quality of life index. By far, the most recognized mechanisms that trigger the progression of this condition are hypersensitivity reactions. As an herbal therapy, Plantago major has been named in several references of Persian traditional medicine with effective properties in ameliorating urticarial symptoms. In this study we aimed to investigate whether Plantago major has the potential to be used clinically in treatment of urticaria according to the current knowledge about the plant effects. Materials and Methods:A combination of "Plantago major" and other terms related to urticarial therapy such as "mast cell", "anti-inflammatory", "prostaglandin", "histamine" and "IgE" that may have possible role in progression or suppression of urticaria was searched in Google Scholar and PubMed as the reference databases.Results:Eleven articles discussing either the direct role of Plantago major or any of its constituents in urticarial related bimolecular pathways were selected.Conclusion:Review of the selected articles indicated that Plantago major can effectively suppress many of hypersensitivity reactions which are also contributors in the pathophysiology of urticaria development. Further clinical trials are needed to prove the efficacy of this herbal treatment.[GMJ.2014;3(2):123-26]
\end{abstract}

Keywords: Urticaria; Antihistamine; Traditional Medicine; Hypothesis

\section{Introduction}

U rticaria, also called hives, is one of several allergic human diseases that presents with sudden outbreak of pale red plaques or wheals and also itchy and raised bumps. Also the disease is usually the result of body reactions to certain foods or medicines, the condition may also exacerbate when patients are exposed to high levels of tension and stress [1].

\section{GMJ}

2013 Galen Medical Journal

Fax: +98 7312227091

PO Box 7461686688

Email:info@gmj.ir
On the other hand, this disabling disease has a significant negative effect on patients' quality of life and may result in psychopathological symptoms, such as anxiety [2]. The allergic related histopathologic changes in urticaria indicate that disrupted molecular pathways in regulation of immune related mechanisms may underlie the hypersensitivity presentations such as dilation of capillaries at the site of reaction. Though the exact cause of reac-

\footnotetext{
Correspondence to:

Mohammad Kamalinejad; School of pharmacy, Shahid

Beheshti University of Medical Sciences.

Telephone Number: (+98) 2188200118

Email Address :mkamalinejad@yahoo.com
} 
tions that lead to these eruptions are not definitely explained, mast cell degradation and the abrupt release of uncontrollable amounts of histamines are the sole known fluctuations that play part in most urticarial syndromes [3, 4]. A variety of therapeutic methods are developed to reduce the degranulation of mast cells and inhibit the vast flow of histamines to blood flow. Cromoglycate mediator stabilizers and immunosuppressive agents are not the drugs of choice either due to side-effects or poor improvement $[5,6]$. Because of lowered CNS entrance in comparison to the first generation, currently, the second generation antihistamines such as Cetirizine are the first line of treatment in urticaria. However, antihistamines are not effective in all types of urticaria. Furthermore, prolonged use of antihistamines is limited in cases with renal and hepatic complications [7]. Considering the restrictions of conventional therapies, we decided to investigate Persian traditional medicine books for possible treatments. From several different herbal medicines, the use of Plantago major has been emphasized in most traditional Persian references. Therefore, in this study we aimed to search for potential properties of Plantago major that can support the clinical use of this plant in treatment of urticaria.

\section{Materials and Methods}

After a review on 5 of the most cited articles on urticaria pathogenesis and therapeutic methods, we selected the following keywords which were repeated in all of the manuscripts: "mast cell", "anti-inflammatory", "prostaglandin" "histamine" and "IgE" [1,2,8-10]. Then, to elucidate the possible relation of Plantago major and the mentioned role players in urticaria outbreak, a combination of these keywords together with the term "Plantago major" was searched in Google scholar and PubMed.

\section{Results}

Based on publication year, paper citation and references to the searched keywords in the body of the articles, among similar and different in vitro and in vivo studies ten manuscripts were selected for review (table-1).

Table 1.articles reviewed in this study with indication to Plantago major or its constituents in suppression of urticaria

\begin{tabular}{lll}
\hline Study & $\begin{array}{l}\text { Plantago major Constituents mentioned } \\
\text { in study }\end{array}$ & $\begin{array}{l}\text { Potential pathways involved in treatment of } \\
\text { urticaria }\end{array}$ \\
\hline Ringbom et al. & Ursolic acid & inhibition of cyclooxygenase-2 \\
Stenholm et al. & ursolic acid, oleanolic acid, $\alpha$-linolenic acid & inhibition of cyclooxygenase-2 \\
Magalhães et al. & oleanolic and ursolic acids & inhibition of cyclooxygenase-2, anti-inflammatory \\
Lien et al. & flavonoids, terpinoids and iridoid glycosides & leukocyte migration, mast cell degranulation \\
Kimata et al. & luteolin, baicalein & IgE mediated mast cell degranulation \\
Ikawati et al. & Plantago major alcoholic extract & histamine release \\
Pourmorad et al. & flavonoids & antioxidant \\
Reina et al. & flavonoids, baicalein & antioxidant \\
$\begin{array}{l}\text { Núñez Guillén } \\
\text { et al. }\end{array}$ & Plantago major aqueous Extract & anti-inflammatory \\
Türel et al. & Plantago major & Hepatoprotective, anti-inflammatory \\
\hline
\end{tabular}




\section{Discussion}

As one of the most known herbs in Plantaginaceae family and a plant of temperate zones, the first reports of therapeutic use of Plantago major dates back to ancient Greek medical treatises in the 1 st century A.D. [11, 12]. With a broad range of biochemical components including phenols, terpenoids and iridoids in both leaves and seeds, current studies have attributed many diverse therapeutic aspects to this plant such as anti-inflammatory, antitumor, antimicrobial and antiviral activities $[13,14]$. Anand et al. have suggested a possible role for cyclooxygenase (COX) 2 inhibitors in the treatment of chronic urticaria [15]. Further, ursolic acid, oleanolic acid and $\alpha$-linolenic acid as three Plantago major compounds have shown to exert inhibitory effects on COX-2 catalyzed prostaglandin production [16-18]. Also, the flavonoid compound of Plantago major, Luteolin, has the ability to suppress leukocyte migration and inhibit mast cell degranulation which all can be considered as anti urticaria treatment strategies [19-21]. An in vivo study of several Indonesian medicinal plants by Ikawati et al. has demonstrated the inhibitory activity of alcoholic extracts of Plantago major leaves on IgE-dependent histamine release [22]. Besides the antioxidant activity of flavonoids such as Baicalein, two studies have shown the anti inflammatory and hepatoprotective effects of this plant in animals which makes the use of Plantago major much more plausible in comparison to antihistamisne especially in patients with hepatic impairment [23-26].

\section{Conclusion}

According to our review on Plantago major and its biochemical constituents in relation to the pathogenesis of urticaria and the hypersensitivity reactions involved in this skin condition, it is evident that this plant can suppress related pathways including mast cell degradation, histamine release and inflammation. Additionally, with regard to the hepatoprotective activates of this herb its use in treatment of urticaria may have less restrictions in comparison to anti histamines. Thus, further studies are needed to explore the clinical efficacy of Plantago major.

\section{Acknowledgments}

This article is drawn from the PHD degree thesis of Mohammad Ali Yazdian entitled "Comparison the Efficacy of Plantago major Aqueous Extract with Cetirizine in Treatment of Acute Urticaria: a Double-blind, Randomized Controlled Trial" from the School of Traditional Medicine, Shahid Beheshti University of Medical Sciences, Tehran, Iran.

\section{References}

1. Kaplan A, Greaves M. Pathogenesis of chronic urticaria. Clin Exp Allergy. 2009;39(6):777-87.

2. Barbosa F, Freitas J, Barbosa A. Chronic idiopathic urticaria and anxiety symptoms. J Health Psychol. 2011;16(7):1038-47.

3. Wedi B, Kapp A. Current position of the role of allergic and non-allergic food hypersensitivity in urticaria. Hautarzt. 2006;57(2):101-7.

4. Katelaris $\mathrm{CH}$, Peake JE. Allergy and the skin: eczema and chronic urticaria. Med J Aust. Medical. 2006;185(9):517.
5. Morgan M, Khan DA. Therapeutic alternatives for chronic urticaria: an evidence-based review, part 2. Ann Allergy Asthma Immunol. 2008;100(6):517-26.

6. Guldbakke KK, Khachemoune A. Etiology, classification, and treatment of urticaria. Cutis. 2007;79(1):41-9.

7. Jáuregui I, Ferrer M, Montoro J, Dávila I, Bartra J, del Cuvillo A, et al. Antihistamines in the treatment of chronic urticaria. J Investig Allergol Clin Immunol. 2007;17(Suppl 2):41-52.

8. Sabroe RA, Greaves MW. The pathogenesis 
of chronic idiopathic urticaria. Arch Dermatol. 1997;133(8):1003-8.

9. Schocket AL, editor. Chronic urticaria: pathophysiology and etiology, or the what and why. Allergy \& Asthma Proceedings; 2006.

10. Lee EE, Maibach HI. Treatment of urticaria. An evidence-based evaluation of antihistamines. Am J Clin Dermatol. 2001;2(1):27-32.

11. Samuelsen AB. The traditional uses, chemical constituents and biological activities of Plantago major L. A review. J Ethnopharmacol. 2000;71(1):1-21.

12. Zubair M, Nybom H, Lindholm C, Rumpunen K. Major polyphenols in aerial organs of greater plantain (Plantago major L.), and effects of drying temperature on polyphenol contents in the leaves. Sci Hortic. 2011;128(4):523-9.

13. Stanisavljević IT, Stojičević SS, Veličković DT, Lazić ML, Veljković VB. Screening the antioxidant and antimicrobial properties of the extracts from plantain (Plantago major L.) leaves. Separation Science and Technology. 2008;43(14):3652-62.

14. Velasco-Lezama R, Tapia-Aguilar R, RománRamos R, Vega-Avila E, Pérez-Gutiérrez MS. Effect of Plantago major on cell proliferation in vitro. J Ethnopharmacol. 2006;103(1):3642.

15. Anand MK, Nelson HS, Dreskin SC. A possible role for cyclooxygenase 2 inhibitors in the treatment of chronic urticaria. J Allergy Clin Immunol. 2003;111(5):1133-6.

16. Ringbom T, Segura L, Noreen Y, Perera P, Bohlin L. Ursolic acid from Plantago major, a selective inhibitor of cyclooxygenase-2 catalyzed prostaglandin biosynthesis. J Nat Prod. 1998;61(10):1212-5.

17. Stenholm $\AA$, Göransson U, Bohlin L. Bioassay-guided Supercritical Fluid Extraction of Cyclooxygenase-2 Inhibiting Substances in Plantago major L. Phytochem Anal. 2013;24(2):176-83.

18. Magalhães WS, Corrêa CM, Alencastro RBd, Nagem TJ. The molecular basis of anti-inflammatory action of the oleanolic and ursolic acids on cyclooxygenase isoforms by docking and molecular dynamics. Química Nova. 2012;35(2):241-8.

19. Lien C, Lean T, Wen C, Mei-Yin C, Chun-Ching L. Immunomodulatory activities of flavonoids, monoterpenoids, triterpinoids, iridoid glycosides and phenolic compounds of Plantago species. Planta med. 2003;69:600-4.

20. Sugita Y, Morita E, Kawamoto H, Horiuchi K, Yamada S, Koro O, et al. Correlation between deposition of immuno-components and infiltration pattern of polymorphonuclear leukocytes in the lesions of chronic urticaria. J dermatol. 2000;27(3):157-62.

21. Kimata M, Shichijo M, Miura T, Serizawa I, Inagaki N, Nagai H. Effects of luteolin, quercetin and baicalein on immunoglobulin E-mediated mediator release from human cultured mast cells. Clin Exp Allergy. 2000;30(4):501-8.

22. Ikawati Z, Wahyuono S, Maeyama K. Screening of several Indonesian medicinal plants for their inhibitory effect on histamine release from RBL-2H3 cells. J Ethnopharmacol. 2001;75(2):249-56.

23. Pourmorad F, Hosseinimehr S, Shahabimajd N. Antioxidant activity, phenol and flavonoid contents of some selected Iranian medicinal plants. African J biotechnol. 2006;5(11).

24. Reina E, Al-Shibani N, Allam E, Gregson K, Kowolik M, Windsor L. The effects of plantago major on the activation of the neutrophil respiratory burst. J Tradit Complement Med. 2013;3(4):268.

25. Núñez Guillén ME, da Silva Emim JA, Souccar C, Lapa AJ. Analgesic and Antiinflammatory Activities of the Aqueous Extract of Plantago major L. Pharmaceutical Biology. 1997;35(2):99-104.

26. Türel I, Özbek H, Erten R, Öner AC, Cengiz N, Yilmaz O. Hepatoprotective and antiinflammatory activities of Plantago major L. Indian J pharmacol. 2009;41(3):120. 